\title{
1 Novel Hydrophilic Matrix System with Non-Uniform 2 Drug Distribution for Zero-Order Release Kinetics
}

3 AUTHORS

4 Matteo Cerea ${ }^{1}$, Alessandra Maroni' ${ }^{1}$, Luca Palugan', Marco Bellini², Anastasia Foppoli1, Alice

5 Melocchi ${ }^{1}$, Lucia Zema ${ }^{1}$, Andrea Gazzaniga ${ }^{1}$

6

$7 \quad$ 1Università degli Studi di Milano

8 Dipartimento di Scienze Farmaceutiche

9 Sezione di Tecnologia e Legislazione Farmaceutiche "Maria Edvige Sangalli"

10 via G. Colombo 71

1120133 Milano, Italy

12

13 2Institut für Pharmazie

14 Freie Universität

15 Kelchstraße, 31

1612169 Berlin, Germany

18 Corresponding author

19 Dr. Anastasia Foppoli, PhD

20 Università degli Studi di Milano

21 Dipartimento di Scienze Farmaceutiche

22 Sezione di Tecnologia e Legislazione Farmaceutiche "Maria Edvige Sangalli"

23 via G. Colombo 71

2420133 Milano, Italy

$25 \quad$ Tel +390250324654

26 email: anastasia.foppoli@unimi.it 
29 Hydrophilic matrices, powder layering, gradient concentration, oral prolonged release, zero-order kinetics, tangential spray rotary fluid bed.

\section{ABSTRACT}

A decrease in the release rate over time is typically encountered when dealing with hydrophilic matrix systems for oral prolonged release due to progressive increase of the distance the drug molecules have to cover to diffuse outwards and reduction of the area of the glassy matrix at the swelling front. In order to solve this issue, a novel formulation approach based on non-uniform distribution of the active ingredient throughout the swellable polymer matrix was proposed and evaluated. Various physical mixtures of polymer (high-viscosity hypromellose) and drug tracer (acetaminophen), having decreasing concentrations of the latter, were applied by powder-layering onto inert core seeds. The resulting gradient matrices showed to possess satisfactory physico-technological characteristics, with spherical shape and consistent thickness of the layers sequentially applied. The non-uniform matrix composition pursued was confirmed by Raman mapping analysis. As compared with a system having uniform distribution of the drug tracer, the multi-layer formulations were proved to enhance linearity of release. The simple design concept, advantageous technique, which involves no solvents nor high-impact drying operations, and the polymeric material of established use make the delivery platform hereby proposed a valuable strategy to improve the performance of hydrophilic matrix systems.

\section{INTRODUCTION}

Tableted matrix systems for oral prolonged release of common cylindrical shape typically display a decrease in the rate of release over time due to progressive increase in the diffusional path the drug has to cover to reach the outer medium and concomitant reduction of the area at the solvent penetration front. Particularly in the case of hydrophilic matrices, an initial burst release may also be observed due to the fraction of drug present on the surface, which is released when the outer polymer particles are not fully swollen yet [1]. Because zeroorder kinetics has long been sought to attain constant drug absorption rate for a predetermined period of time, thus providing the patient with constant drug levels between two successive doses, several attempts to address these issues have been reported in the literature. The resulting systems are intended to reach the goal of a linear release pattern by the use of different strategies, which may involve mechanical restriction of swelling, application of partial coatings and/or design of modified geometries [2-10]. Overall, these approaches would be aimed at restraining the gradual extension of the diffusion path or progressively broadening the area at the solvent penetration front within the matrix.

In addition, gradient systems, wherein the drug concentration increases from the outside towards the inside, have been described [11-18]. Such systems may be obtained by controlled extraction processes, coating/layering techniques or, alternatively, emerging fabrication methods, such as 3D printing and 
electrostatic deposition, that are still poorly exploited in the pharmaceutical field [19-23]. Coating is mainly based on liquid vehicles that not only require time- and energy-consuming drying phases, but also may bring about stability issues. In this respect, aqueous spray-coating would especially be critical when using hydrophilic polymers tending to generate too high viscosities and involving the use of diluted solutions [24,25]. Notably, powder-layering, wherein coating materials are directly layered as solids onto inert starting cores, may overcome most technical issues connected with liquid-based processes [26,27]. For these reasons, it was deemed to be an alternative technique that could advantageously be exploited for manufacturing of hydrophilic gradient matrices.

Accordingly, the aim of the present study was to $i$ ) design a hydrophilic matrix system with non-uniform drug distribution (Non-Uniform Drug Distribution Matrix, NUDDMat) for prolonged release, intended to ultimately provide zero-order kinetics, ii) study the feasibility of powder-layering in the relevant manufacturing through application of successive layers having decreasing drug concentrations, and iii) evaluate the in vitro performance of the resulting prototypes.

\section{MATERIALS AND METHODS}

\section{MATERIALS}

Acetaminophen fine powder British Pharmacopoeia (AAP, CFM, Italy) (water solubility $14.8 \mathrm{mg} / \mathrm{mL}$, true density $1.13 \mathrm{~g} / \mathrm{mL}$ [28-30]) was used as the tracer and high viscosity hypromellose (HPMC, Methocel ${ }^{\circledR} \mathrm{K} 15 \mathrm{M}$, Colorcon, Italy) (apparent viscosity, $2 \%$ in water at $20{ }^{\circ} \mathrm{C} 6138-9030 \mathrm{mPa} * \mathrm{~s}$, USP substitution type 2208, true density $1.32 \mathrm{~g} / \mathrm{mL}$ [31-33]) was selected as the hydrophilic swellable polymer. Lactose (Carlo Erba, Italy, true density $1.50 \mathrm{~g} / \mathrm{mL}$ [34]) and dibasic calcium phosphate dihydrate (DCP, Emcompress ${ }^{\circledR}$, JRS, Germany, true density $2.21 \mathrm{~g} / \mathrm{mL}$ [35]) were evaluated as soluble and insoluble diluents. Fumed silica (Aerosil ${ }^{\circledR} 200$, Evonik, Germany) was added as a glidant to the powder mixtures, and povidone (PVP, Kollidon ${ }^{\circledR} 30$, BASF, Germany, true density $1.11 \mathrm{~g} / \mathrm{mL}$ [36]) was used as the binder in aqueous solution.

Nonpareil microcrystalline cellulose (MCC) pellets having nominal diameter of $850 \mu \mathrm{m}$ were chosen as the starting cores (Cellets ${ }^{\circledR} 700$, Pharmatrans-Sanaq, Switzerland, true density $1.46 \mathrm{~g} / \mathrm{L}$ [37]).

\section{METHODS}

\section{Manufacturing of tableted and layered units}

According to formulas set up during the experimental work, reported in the Results and Discussion section, powder blends to be tableted or layered were obtained by mixing in Turbula ${ }^{\circledR}$ (Willy A. Bachofen, Switzerland) at $24 \mathrm{rpm}$ for $20 \mathrm{~min}$.

Tableting was performed by a rotary tablet press (mod. AM8S, Officine Meccaniche Ronchi, Italy) equipped with $4 \mathrm{~mm}$ diameter concave punches ( $4 \mathrm{~mm}$ curvature radius) at $7 \mathrm{kN}$ compression force (batch size $50 \mathrm{~g}$ ). The nominal weight of matrices was $45 \mathrm{mg}$. 
Powder layering was performed by a fluid bed (GPGC 1.1, Glatt, Germany) equipped with rotor insert. The operating conditions set up were as follows: nozzle port size $1.2 \mathrm{~mm}$, air temperature in $30{ }^{\circ} \mathrm{C}$, product temperature $26-28{ }^{\circ} \mathrm{C}$, air temperature out $23{ }^{\circ} \mathrm{C}$, air flow $70 \mathrm{~m}^{3} / \mathrm{h}$, nebulization air pressure 2 bar, product pressure $1100 \mathrm{~Pa}$, disk rotation speed $700 \mathrm{rpm}$, liquid binder feeding rate $12 \mathrm{~g} / \mathrm{min}$, powder feeder rate 20 $\mathrm{g} / \mathrm{min}$. For any layering step, $500 \mathrm{~g}$ of starting substrate was loaded into the processing chamber. Powder addition was accomplished via forced powder feeder while spraying a binding solution onto preheated substrate cores. The diameter and weight of the coated units were regularly checked in-process by withdrawing samples $(\mathrm{n}=100)$ at prefixed time intervals. After each layering step, a post-processing drying phase was performed at inlet air temperature of $60{ }^{\circ} \mathrm{C}$ for $30 \mathrm{~min}$. Process yield was calculated as the percentage weight ratio between the layered units and the employed materials (seeds, layering powders, binder).

\section{Physico-technological characterization of tableted and layered units}

Tableted $(n=20)$ and layered $(n=100)$ units were checked for weight and for thickness and/or diameter by a precision calliper (CD 150, Mitutoyo, Italy).

The aspect ratio was calculated dividing the maximum by the minimum diameter of each unit, as measured by digital microscope (Dino Lite Pro AM 413T, Italeco, Italy) (n=20).

Friability was assessed according to Eur. Ph. 9.2 by a friabilometer (mod. EF-2, Electrolab, Italy) rotating at $25 \mathrm{rpm}$ for $4 \mathrm{~min} .6 .5 \mathrm{~g}$ of units was used for the measurements. The data obtained were expressed as loss-onweight percentages.

Crushing strenght $(\mathrm{n}=10)$ was measured by the equipment reported in Eur. Ph. 9.2 (mod. T3, Erweka, Germany).

Porosity $(E)$ of units, expressed as percentage, was calculated according to the equation:

$$
E(\%)=\left[1-\frac{V_{t}}{V_{b}}\right] \cdot 100
$$

where $\mathrm{Vt}$ is the true volume and $\mathrm{Vb}$ is the mean bulk volume.

Cross-section morphology was analyzed by a scanning electron microscope (SEM; Sigma, Carl Zeiss, Germany). Samples were gold-sputtered using a plasma evaporator under vacuum, and photomicrographs were acquired at an accelerated voltage of $10 \mathrm{kV}$ at differing magnifications.

\section{Release testing of tableted and layered units and data analysis}

Samples corresponding to an overall amount of $50 \mathrm{mg}$ of AAP were tested for release by a Eur. Ph. 9.2 dissolution apparatus (mod. 2100B, Distek Italia, Italy) equipped with rotating baskets using $900 \mathrm{~mL}$ of purified water at $37 \pm 0.5{ }^{\circ} \mathrm{C}$ stirred at $100 \mathrm{rpm}(\mathrm{n}=3)$. AAP was assayed by spectrophotometer at $243 \mathrm{~nm}$ (Lambda 25, Perkin Elmer Italia, Italy) after verifying agreement of results with a HPLC method according to 
USP 40-NF 35 [38]. Samples of medium were collected at 4 selected time points $(0.5-70.0 \mu \mathrm{g} / \mathrm{mL}$ concentration range) during the release test. Each sample was analyzed $(n=6)$ by both HPLC (column: $\mathrm{C}_{18}$ at $40{ }^{\circ} \mathrm{C}$, mobile phase: deionized water for HPLC/acetonitrile/perchloric acid 3000:1000:3, V/V/V, at pH 2.4, flow rate: $1.2 \mathrm{~mL} / \mathrm{min}$, detection: $\mathrm{UV}$ at $248 \mathrm{~nm}$, injection volume: $40 \mu \mathrm{L}$ ) and $\mathrm{UV}$ using freshly prepared standard solutions as a reference. The 95\% confidence intervals for the differences in the measured concentration means (UV minus HPLC) fell in the $\pm 2 \%$ range with respect to the HPLC mean, in compliance with typical acceptance criteria [39]. For statistical comparison of release profiles, similarity factor $\mathrm{f}_{2}$ was applied. $f_{2} \geq 50$ (50-100) indicated similarity [40].

142 Release data from UDDMat systems were analyzed according to the equation:

$$
\frac{M_{t}}{M_{\infty}}=k t^{n}
$$

where $M_{t} / M_{\infty}$ is the drug fraction released at time $t, n$ exponent indicates the mechanism/kinetics of release and $k$ is a constant incorporating structural and geometrical characteristics of the matrix. Analysis of data was performed in the portion of the curve where $M_{t} / M_{\infty}<0.60$, and confidence intervals (c.i.) at $95 \%$ were calculated [41-43].

Equation (2) was also used to fit release data from NUDDMat systems. In this case, the portion of the curve wherein $\mathrm{M}_{t} / \mathrm{M}_{\infty}<0.90$ was analyzed, and $n$ values were used for descriptive purposes only to highlight progressive shift toward linearity. When $n$ was $=1$, the units were considered, from a merely phenomenological point of view, to behave as a zero-order release system, and values $<0.5$ and $>1$ could also be taken into account.

The extent of linearity of release profiles was evaluated through the Durbin-Watson statistics, which enables to identify zero-order portions in each curve $[42,44,45]$. The release data were analyzed at time intervals of 1 h, between 0.05 and 0.90 fractions released (90\% c.i.).

\section{Raman mapping analysis}

Raman mapping analysis was performed on cross-sectioned units using a confocal microscope XploRA Plus ${ }^{\mathrm{TM}}$ (HORIBA Italia, Italy), under the following conditions: acquisition time $0.6 \mathrm{~s}$, laser $785 \mathrm{~nm}$, grating 600 grooves/mm, objective 100x, spatial step size $40 \mu \mathrm{m}$, measured area $100 \times 100$ points (pixels) covering an area of $4000 \times 4000 \mu \mathrm{m}$ [46]. After acquiring Raman spectra of AAP, HPMC and DCP, the relevant identification was accomplished by a correlation algorithm through KnowItAll ${ }^{\mathrm{TM}}$ spectral database (HORIBA Italia, Italy). Hyperspectral mapping of the cross-sectioned systems was carried out moving the laser across the surface and acquiring a spectrum for each point. False color maps were generated after calibration of peaks related to the 3 components considered: green was assigned to AAP, blue to HPMC (or cellulose) and red to DCP. Maps were created by applying fast mapping mode on SWIFT ${ }^{\mathrm{TM}}$ accessory to reduce acquisition time while maintaining high resolution of spectra. The intensity ratio between the colors assigned to AAP and HPMC (AAP/HPMC ratio) was calculated. The AAP/HPMC ratio was associated to a false color (turquoise), and 2D 
as well as 3D intensity maps were generated. Profiles of AAP/HPMC ratio versus diameter of the units were

171 also constructed.

173 RESULTS AND DISCUSSION

175 Uniform Drug Distribution Matrix Systems

176 With the aim of overcoming typical issues involved by hydrophilic matrices, namely the initial burst release

177 and gradual decrease in the release rate, an alternative strategy was explored for the relevant formulation and 178 manufacturing. Various swellable polymers have been proposed in the literature as matrix-forming agents, 179 such as hypromellose (HPMC), hydroxypropylcellulose (HPC) and polyethylene oxide (PEO) [1]. HPMC is 180 especially popular for the long-established regulatory acceptability and availability of different grades (degree 181 of substitution, molecular weight, particle size). Methoce ${ }^{\circledR} \mathrm{K} 15 \mathrm{M}$, a high viscosity HPMC, was selected for 182 this study, and AAP was employed as an analytical tracer. Using a polymer percentage that could be considered 183 sufficient for formation of a non-disintegrating swellable hydrophilic matrix, systems based on a 30:70 184 HPMC/AAP mixture were first investigated (Table 1). Such systems were manufactured by either 185 conventional tableting (UDDMat T30) or powder layering (UDDMat P30) in order to assess the application 186 potential of the latter technique. For a successful outcome, powder layering requires the use of powders having 187 adequate flowing and adhesion properties. While the flowability of this blend proved to be suitable not only 188 for tableting but also for powder layering, the particle adhesion needed to be promoted by spraying a binder 189 solution for effective deposition. PVP, HPC and HPMC have been reported to be the most commonly used 190 binding agents for powder layering [47,48]. In particular, a 5\% PVP (Kollidon ${ }^{\circledR} 30$ ) solution was used, which 191 displayed satisfactory binding properties and suitable viscosity for nebulization. 
Table 1: tracer/polymer/diluent percentage composition of the different powder blends to be tableted or layered ${ }^{\circ}$

\begin{tabular}{|c|c|c|c|c|c|c|}
\hline \multicolumn{2}{|c|}{ System code } & \multirow{2}{*}{$\begin{array}{c}\text { Layer } \\
-\end{array}$} & \multirow{2}{*}{$\frac{\text { AAP }(\%)}{70}$} & \multirow{2}{*}{$\frac{\text { HPMC }(\%)}{30}$} & \multirow{2}{*}{$\begin{array}{c}\text { Lactose (\%) } \\
-\end{array}$} & \multirow{2}{*}{$\frac{\text { DCP }(\%)}{-}$} \\
\hline UDDMat & T30 & & & & & \\
\hline Distribution Matrices) & P30 & - & 70 & 30 & - & - \\
\hline \multirow{17}{*}{$\begin{array}{c}\text { NUDDMat } \\
\text { (Non-uniform Drug } \\
\text { Distribution Matrices) }\end{array}$} & & $1^{\text {st }}$ & 80 & 20 & - & - \\
\hline & & $2^{\text {nd }}$ & 65 & 35 & - & - \\
\hline & G20-80 & $3^{\text {rd }}$ & 50 & 50 & - & - \\
\hline & & $4^{\text {th }}$ & 35 & 65 & - & - \\
\hline & & $5^{\text {th }}$ & 20 & 80 & - & - \\
\hline & G20-100 \# & $5^{\text {th }}$ & - & 100 & - & - \\
\hline & G20-100/2 \#* & $5^{\text {th }}$ & - & 100 & & \\
\hline & & $1^{\text {st }}$ & 65 & 30 & 5 & - \\
\hline & & $2^{\text {nd }}$ & 50 & 30 & 20 & - \\
\hline & G30-30L & $3^{\text {rd }}$ & 35 & 30 & 35 & - \\
\hline & & $4^{\text {th }}$ & 20 & 30 & 50 & - \\
\hline & & $5^{\text {th }}$ & - & 30 & 70 & - \\
\hline & & $1^{\text {st }}$ & 65 & 30 & - & 5 \\
\hline & & $2^{\text {nd }}$ & 50 & 30 & - & 20 \\
\hline & G30-30D & $3^{\text {rd }}$ & 35 & 30 & - & 35 \\
\hline & & $4^{\text {th }}$ & 20 & 30 & - & 50 \\
\hline & & $5^{\text {th }}$ & - & 30 & - & 70 \\
\hline
\end{tabular}

${ }^{\circ} 0.75 \%$ of fumed silica was added to each powder formulation

\# composition of $1^{\text {st-4th }}$ layers is the same as in NUDDMat G20-80

209 The powder layering process was performed by rotary fluid bed starting from $850 \mu \mathrm{m} \mathrm{MCC}$ seeds. Preliminary 210 trials allowed the operating parameters to be adjusted. An efficient process was finally set up and a yield of $21188.7 \%$ was reached. Powder layering was continued until the diameter of the units was approximately of $4 \mathrm{~mm}$ 212 as in the reference tableted matrices. The volume occupied by the starting seed was considered negligible. 213 Systems having an average weight of $45.75 \mathrm{mg} \pm 0.74$ s.d. and friability of $0.3 \%$ were obtained, which turned 214 out comparable with tablets (weight $47.11 \mathrm{mg} \pm 0.29$ s.d., friability $0.2 \%$ ). Crushing strenght of layered 215 UDDMat units was significantly higher than that of tablets, i.e. $55 \mathrm{~N} \pm 8$ s.d. versus $24 \mathrm{~N} \pm 5$ s.d. $(\mathrm{p}<0.01)$, 216 respectively, probably owing to the use of an aqueous binder solution for layering. Interestingly, the two 217 different techniques led to very similar porosity for tablets and layered units: $4.8 \%$ and $4.9 \%$, respectively. Porosity, however, is known to only poorly influence the performance of hydrophilic matrices [49]. 
221 The basket dissolution apparatus was employed for release tests since the layered systems exhibited a certain 222 tendency to buoyancy. The use of sinkers with paddles was discarded because of major drawbacks in terms of swelling constraints. The systems having uniform drug distribution, irrespective of whether they had been manufactured by tableting or powder layering, showed a progressive decrease in the release rate over time after a slight burst release phase (Figure 1). Indeed, when using equation (2), $n$ of 0.832 (0.061 c.i.) and 0.775 ( 0.056 c.i.) obtained from tableted and layered matrices, respectively, highlighted non-fickian anomalous release behaviour. The difference observed in $n$ values between almost cylindrical compressed and spherical layered matrices could be ascribed to the diverse shape, being included in the range predicted for cylinders and spheres [50]. An almost superimposable release pattern was seen $\left(f_{2}=68.75\right)$, indicating that neither the use of powder layering for this particular application, nor the composition change required, i.e. the addition of PVP in the binding solution, would significantly affect the release performance.

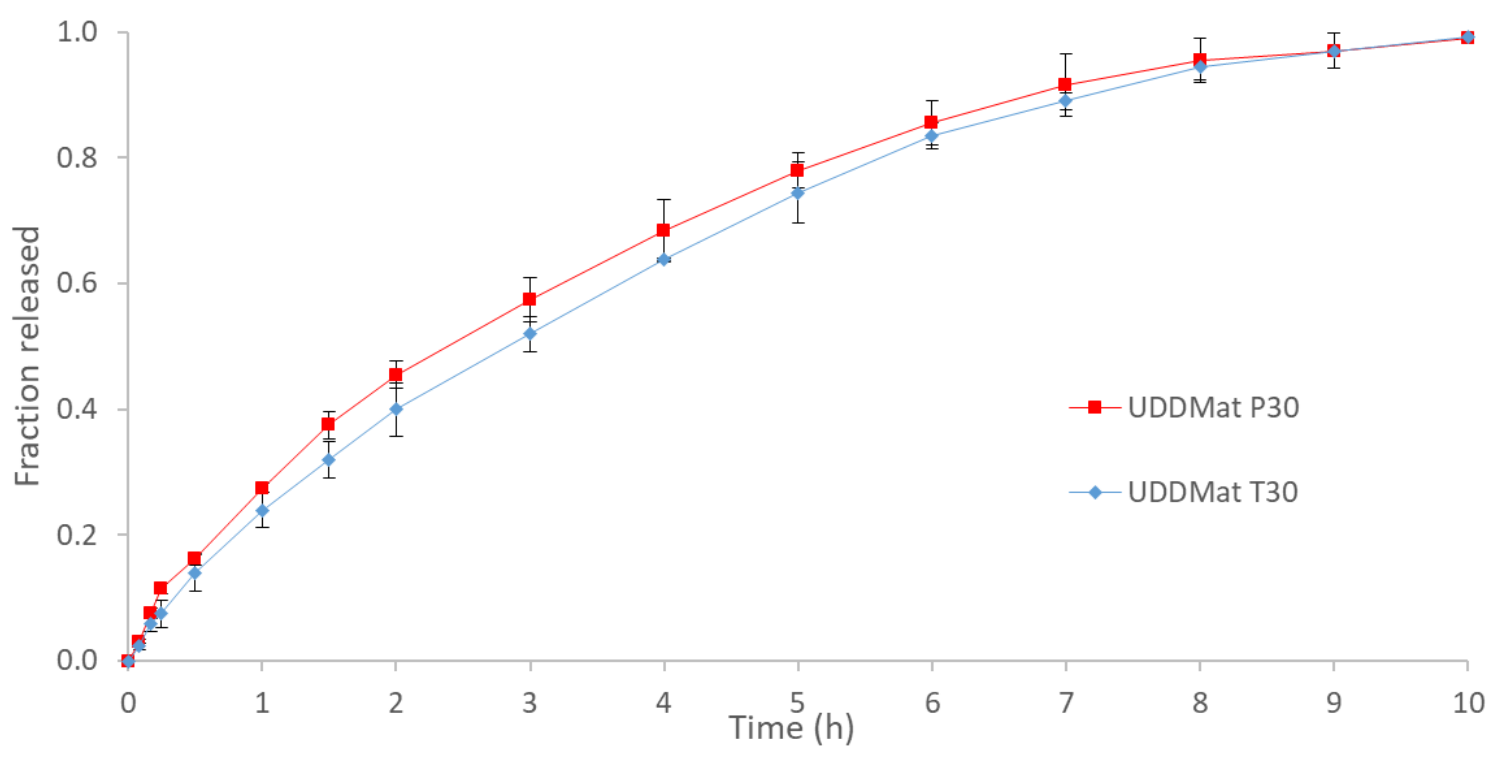

Figure 1: release profiles from tableted (UDDMat T30) and layered (UDDMat P30) matrices with uniform drug distribution. Vertical bars represent standard deviations.

Non-Uniform Drug Distribution Matrix Systems

238 Powder layering was thus undertaken for the manufacturing of the Non-Uniform Drug Distribution Matrix 239 (NUDDMat). This system was designed to include 5 overlaid layers differing in drug concentration, which 240 decreased between contiguous layers from the inside towards the outside of the matrix according to a 241 descending staircase function, ultimately tending to an apparent overall linear mode [13,17]. In Figure 2, a general outline of the NUDDMat system and layer-by-layer representation of the relevant theoretical drug concentration profile are presented. The nominal thickness of each layer was $315 \mu \mathrm{m}$ aiming at a final diameter 
244 of $4 \mathrm{~mm}$, as in the previous systems having uniform drug distribution, including the diameter of the core (mean 245 diameter $850 \mu \mathrm{m})$.

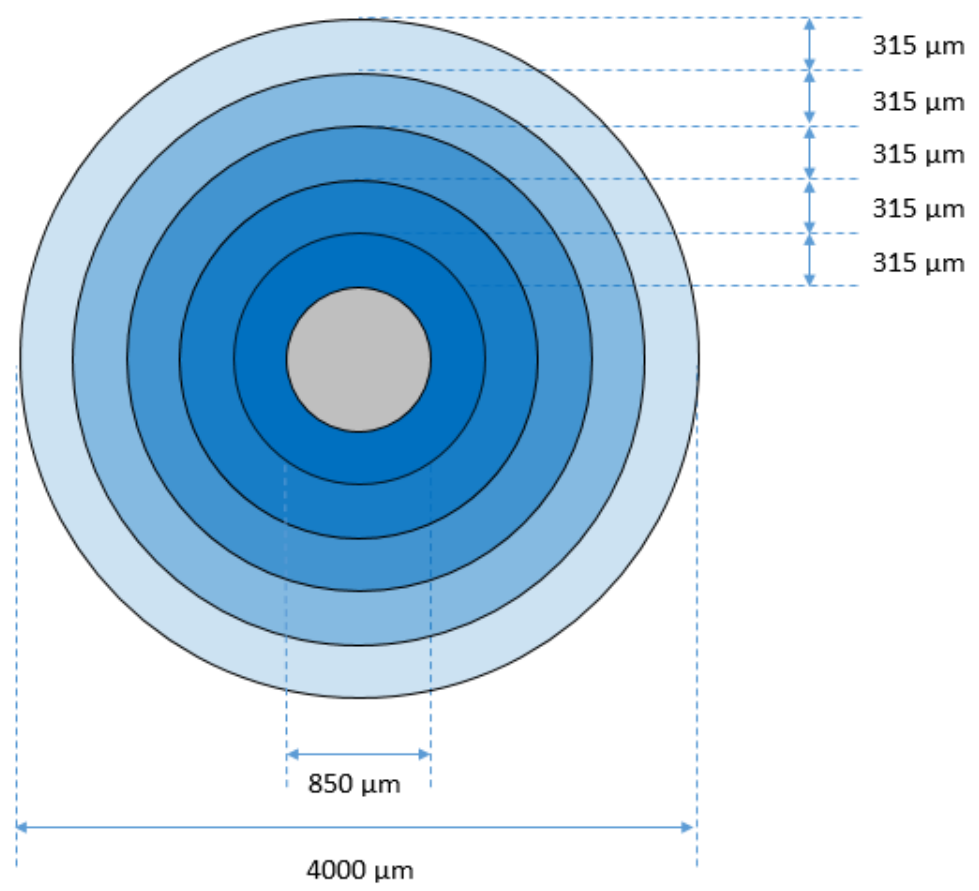

b)

Figure 2: a) NUDDMat design concept (drug tracer concentration is indicated by color intensity); b) theoretical tracer concentration pattern along the radius (the dotted line indicates the overall decreasing trend of concentration).

G20-80 was the first NUDDMat system prepared, according to the above-illustrated design concept. The

260 1, taking the need for binder and glidant into account. PVP and fumed silica were maintained at $3.00 \%$ and at $2610.72 \%$, respectively. 
In particular, the percentage of PVP was calculated based on a 5:3 weight ratio between layered powder and $5 \% \mathrm{w} / \mathrm{w}$ binding solution. The amount of materials required for each layer was calculated based on the true density of the formulation components by assuming a layer porosity of $5 \%$ (Table 2 ).

Table 2: nominal diameter, volume, weight, drug content and drug concentration, cumulative and for each layer, in

NUDDMat G20-80 system

\begin{tabular}{|c|c|c|c|c|c|c|c|c|}
\hline Unit & $\begin{array}{l}\text { Cumulative } \\
\text { diameter } \\
(\mathrm{mm})\end{array}$ & $\begin{array}{c}\text { Layer } \\
\text { volume } \\
\left(\mathrm{mm}^{3}\right)\end{array}$ & $\begin{array}{c}\text { Cumulative } \\
\text { volume } \\
\left(\mathrm{mm}^{3}\right)\end{array}$ & $\begin{array}{c}\text { Layer } \\
\text { weight } \\
(\mathrm{mg})\end{array}$ & $\begin{array}{c}\text { Cumulative } \\
\text { weight } \\
(\mathrm{mg})\end{array}$ & $\begin{array}{c}\text { Layer } \\
\text { drug content } \\
(\mathrm{mg})\end{array}$ & $\begin{array}{c}\text { Cumulative } \\
\text { drug content } \\
(\mathrm{mg})\end{array}$ & $\begin{array}{c}\text { Layer drug } \\
\text { concentration } \\
\left(\mathrm{mg} / \mathrm{mm}^{3}\right)\end{array}$ \\
\hline Core seed & 0.85 & - & 0.32 & - & 0.50 & - & - & - \\
\hline 1-layer & 1.48 & 1.38 & 1.70 & 1.50 & 2.00 & 1.16 & 1.16 & 0.84 \\
\hline 2-layer & 2.11 & 3.22 & 4.92 & 3.55 & 5.55 & 2.22 & 3.38 & 0.69 \\
\hline 3-layer & 2.74 & 5.85 & 10.77 & 6.50 & 12.05 & 3.13 & 6.51 & 0.53 \\
\hline 4-layer & 3.37 & 9.26 & 20.03 & 10.39 & 22.43 & 3.50 & 10.01 & 0.38 \\
\hline 5-layer & 4.00 & 13.46 & 33.49 & 15.22 & 37.66 & 2.93 & 12.94 & 0.22 \\
\hline
\end{tabular}

It ensues that the theoretical total amount of drug tracer for each unit was approximately $13 \mathrm{mg}$, corresponding to $34.4 \%$ of the overall mass, the weight percentage of the starting seed being about $1.3 \%$.

Due to their differing compositions, the powder mixtures employed for the manufacturing of NUDDMat G2080 exhibited diverse flow properties ranging from very poor to poor according to Eur. Ph. 9.2 classification (compressibility index from $32 \%$ of the inner layer formulation to $28 \%$ of the outer one). Nevertheless, the powder feeding device in use allowed all mixtures to be loaded into the processing chamber of the fluid bed at a sufficiently reproducible rate consistent with continuous mode of dosing, and no formulation changes were needed. Powder adhesion onto the cores was expectedly enhanced with increasing percentage amounts of polymer in the mixtures. However, a 5\% PVP solution, which was sprayed when layering the 30:70 HPMC:AAP mixture within the preparation of UDDMat P30, proved also effective with the most critical 20:80 blend, i.e. the formulation of the innermost layer. On the other hand, although simple spraying of water would have been possible with the mixtures having higher HPMC content, the same type and amount of binder solution was utilized for consistency in all powder layering steps. During the whole process, a 5:3 weight ratio was maintained between powder dosing rate $(20 \mathrm{~g} / \mathrm{min})$ and binder spraying rate $(12 \mathrm{~g} / \mathrm{min})$, so that the percentage of PVP could be the same in all layers.

285 The powder layering process was run in 5 successive steps, each corresponding to a single layer, starting from 286 a $500 \mathrm{~g}$ load of either inert seeds or intermediate layered units. By assuming a yield of 90\%, the amount of 287 powder formulation to be applied for each layer was calculated so that the desired $315 \mu \mathrm{m}$ increase in thickness 
could be reached. When the whole quantity of powder blend needed per layer was loaded, the resulting layered units underwent a drying phase and were then checked for weight, diameter, porosity, crushing strenght, friability and aspect ratio (Table 3). The increases in weight and diameter were close to the expected values, although slightly higher data were generally obtained because of the process yield being greater then assumed. Porosity values turned out slighly higher than predicted with respect to those exhibited by UDDMat systems. The overall mechanical characteristics were proved satisfactory. Indeed, friability was approximately $0.4 \%$, and crushing strenght was remarkably higher than in the case of both tableted and layered UDDMat systems having 30\% of HPMC. The aspect ratio was around 1 thus indicating that NUDDMat G20-80 system had spherical shape, as confirmed by visual inspection (Figure 3).

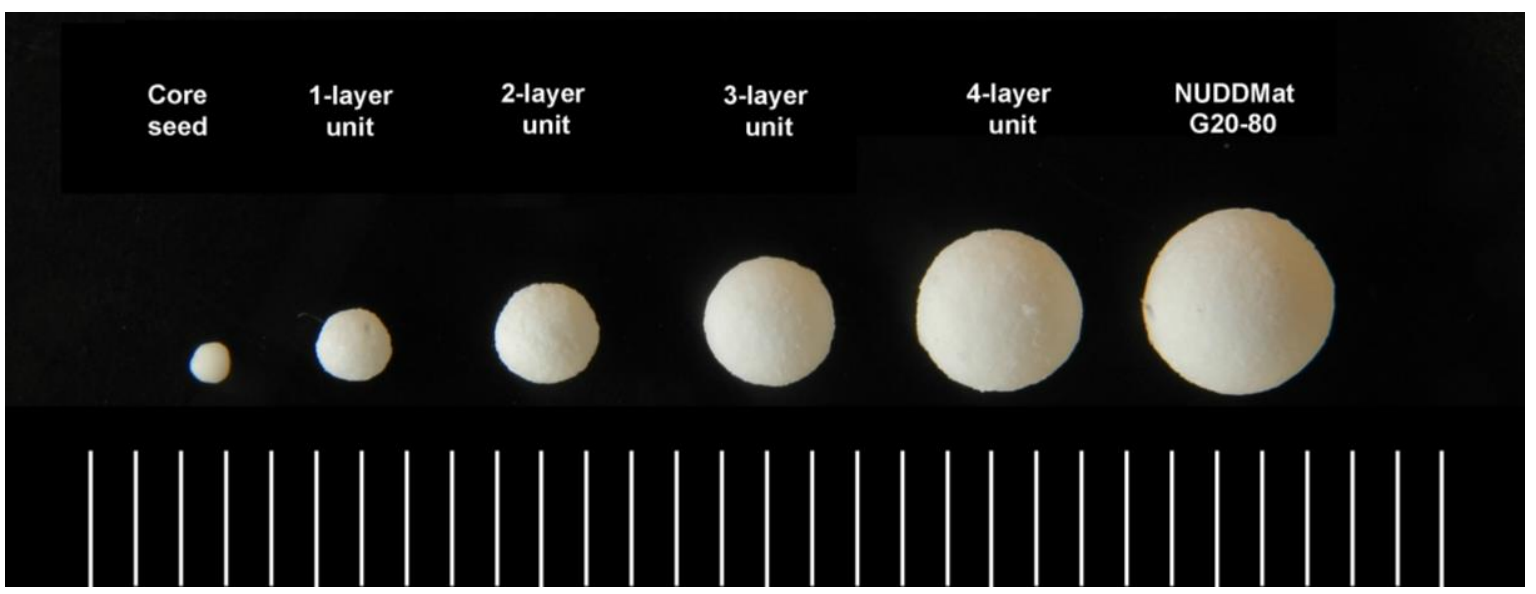

Figure 3: units ranging from the core seed to the final NUDDMat G20-80 system (scale in millimeters).

The expected internal onion-like structure of NUDDMat G20-80, generated by discontinuous processing through application of powders with differing compositions, can barely be distinguished from SEM photomicrographs of a cross-sectioned unit (Figure 4). The dimple that is visible in the center of the system was left by the core seed, which was removed upon sectioning. 


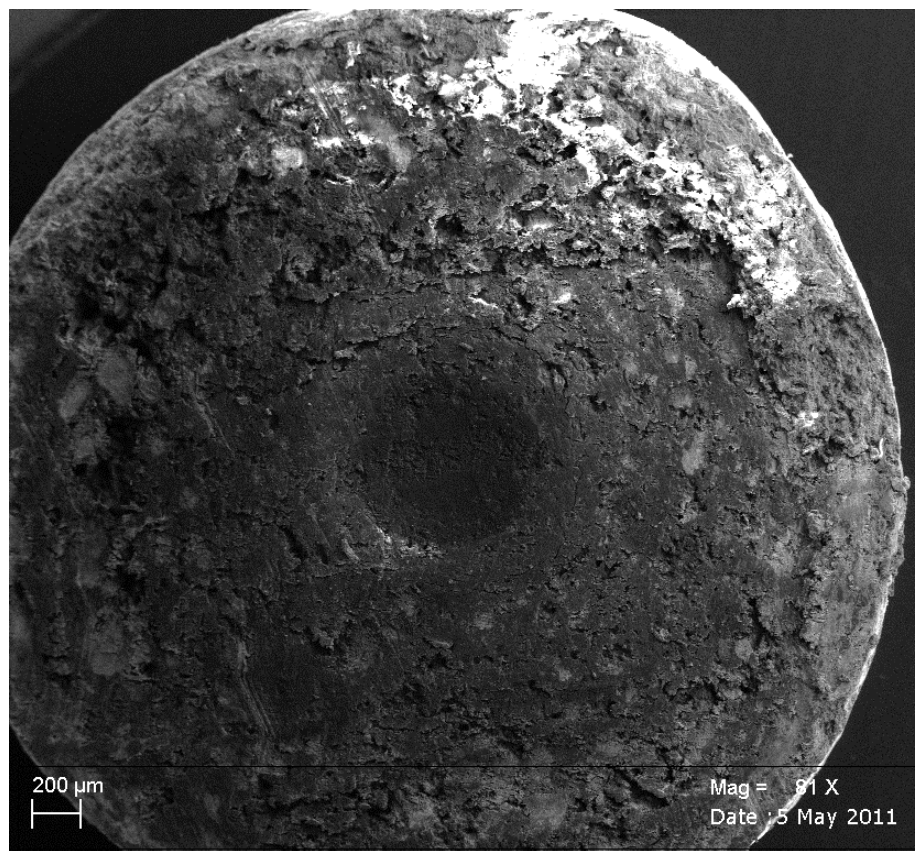

309 The release profiles of the final NUDDMat G20-80 system and of the relevant intermediate units having 1 to 3104 layers are reported in Figure 5.

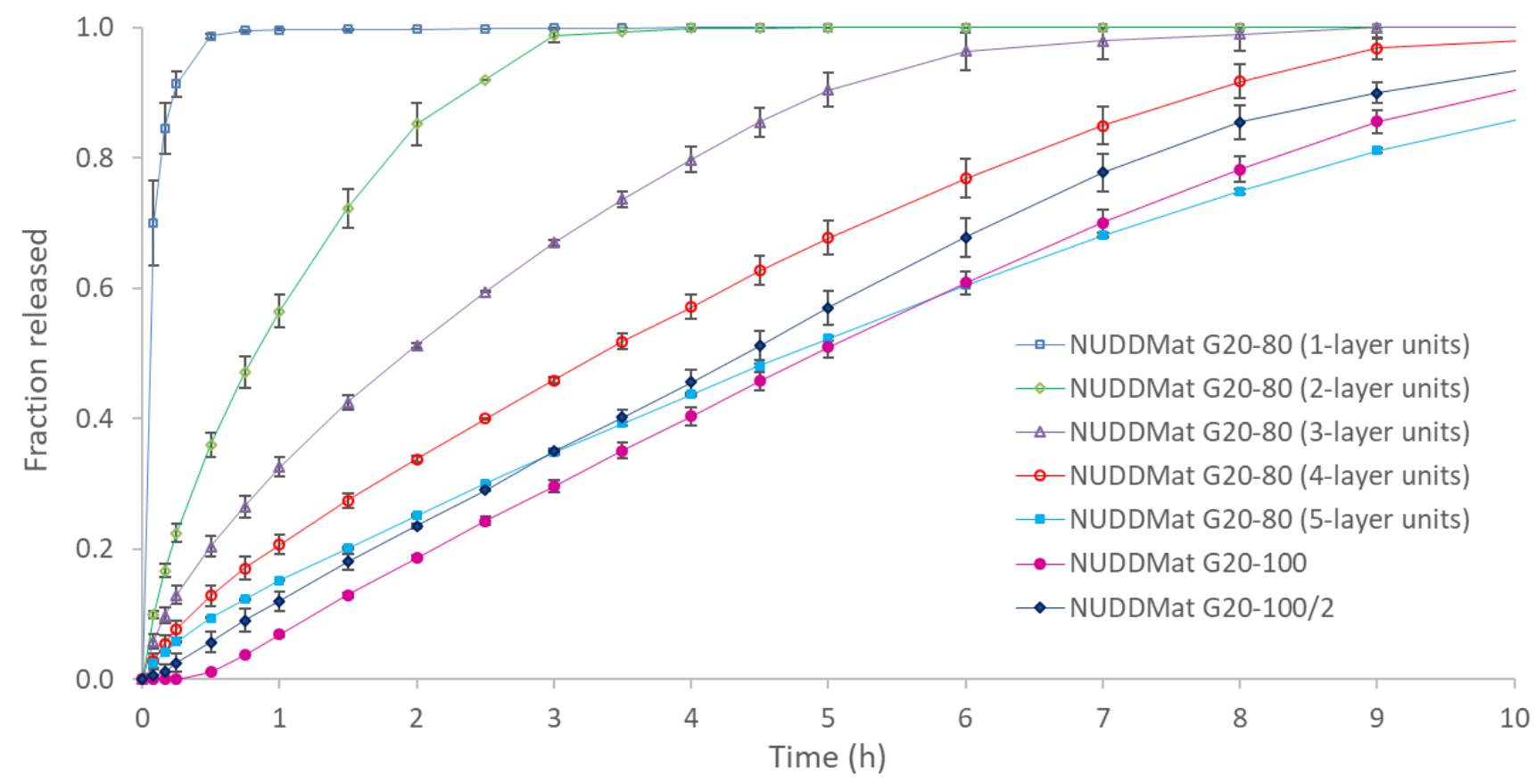


315 The $n$ values resulting from data processing by exponential equation (2), here used for merely descriptive 316 purposes, progressively increased thus pointing out a shift of the curves towards linearity (Table 4).

317 One-layer units released the drug tracer rapidly due to a limited percentage amount of polymer. Indeed, the 318 matrix structure could not withstand rapid erosion/disintegration, and the $n$ value provided no meaningful 319 information. On the other hand, with units having 2 or more layers, the formation of a swollen gel barrier was 320 soon evident during the test. The rate of release decreased as a function of the number of layers. $n$ values 321 showed an increasing trend, thus indicating a shift towards linearity. Accordingly, the linear portion of the release curve from NUDDMat G20-80 (5 layers) ranged from 0.15 to 0.44 of fraction released, and the relevant time frame was $1 \div 4 \mathrm{~h}$, as assessed by Durbin-Watson statistics. However, an initial phase of higher release rate could still be noticed.

Aiming to overcome occurrence of this slight burst, a modified five-layer system, NUDDMat G20-100, was prepared and evaluated. In particular, the $5^{\text {th }}$ layer of NUDDMat G20-100 was devoid of drug tracer, which was replaced by a corresponding amount of HPMC (Table 1). The physical technological characteristics were still satisfactory, and the outermost layer composed of sole polymer turned out effective in suppressing the undesired burst effect (Table 3, Figure 5). However, it brought about a lag time before the onset of release, as testified by $n$ rising up to 1.121 (Table 4). Therefore, the thickness of the outer layer was halved in an attempt to reduce the lag time (NUDDMat G20-100/2), with no impact on mechanical properties was observed (Table 3). This system proved useful to solve the lag phase issue and able to yield practically linear release curves ( $n=0.993$ ) by preventing any burst effect (Figure 5, Table 4). Based on Durbin-Watson statistics, the profile turned out linear from 0.12 to 0.79 of fraction released, between 1 to $7 \mathrm{~h}$. Interestingly, the onion-like structure of NUDDMat systems, which was expected to present concentration steps according to the descending staircase function conceived, was apparently not reflected in the release patterns obtained. Indeed, such steps might have been smoothed through partial migration of the drug tracer upon exposure to the aqueous binding solution during preparation and/or inward diffusion of the aqueous medium during release tests.

Because the concentration of HPMC in the NUDDMat systems described so far, of approximately $60 \%$, largely exceeded that usually employed for prolonged-release matrices, the possibility of reducing the overall amount of polymer was explored. Accordingly, systems with polymer content of 30\% in all 5 layers, each of $315 \mu \mathrm{m}$, were conceived, wherein $i$ ) the amount of HPMC was increased in the first layer to the detriment of the drug tracer, ii) the amount of HPMC was decreased in the other 4 layers through replacement with an insoluble (DCP) and a soluble (lactose) excipient, and iii) no drug was loaded into the outer layer. The resulting formulations were named NUDDMat G30-30D and G30-30L, respectively, and the relevant compositions are reported in Table 1. In both cases, slightly improved powder layering processing was noticed, possibly due to the better flowability properties of the diluents as compared with HPMC. The lower amount of polymer was reflected in reduced crushing strenghts, which, however, still remained satisfactory (Table 3). 

system (Figure 6).

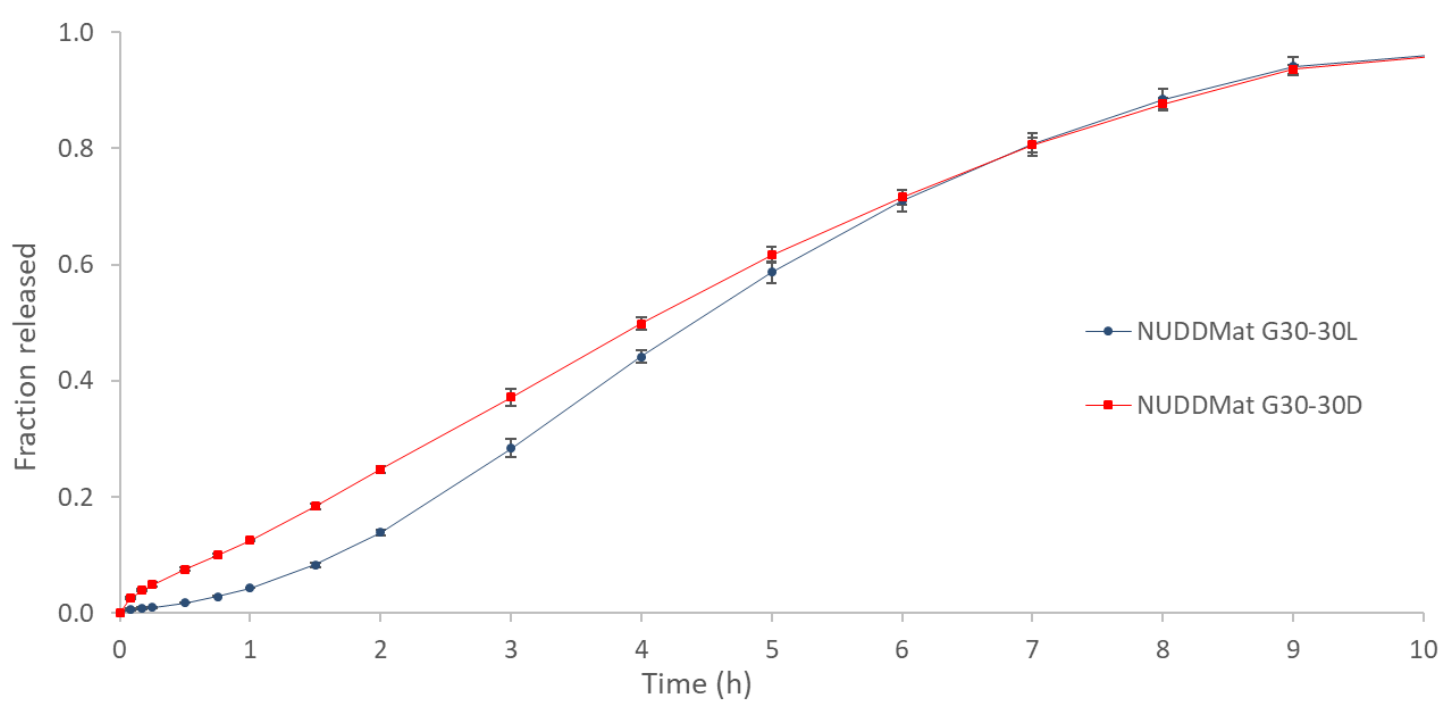

Figure 6: release profiles from NUDDMat G30-30L and NUDDMat G30-30D systems.

357 A slight burst was observed in the relevant curve, even though no drug was contained in the outer layer. This 358 could be attributed to destructuring of the swollen outer polymer layer caused by the dispersed particles of 359 insoluble DCP, here present at the highest concentation of $70 \%$. In contrast, release of the drug tracer from 360 NUDDMat G30-30L was slowed down in the first hour of testing, probably because of soluble lactose bringing 361 about fast formation of an external gel barrier responsible for initially slow diffusion. As shown before for 362 G20-100, this issue could be counteracted by simply reducing the outer layer thickness.

363 Interestingly, data from NUDDMat G30-30D and G30-30L indicated that partial replacement of the polymer 364 would not impair the overall release control. 
Table 3: weight, size, porosity, aspect ratio, crushing strenght and friability of NUDDMat units

\begin{tabular}{ccccccc}
\hline NUDDMat unit & $\begin{array}{c}\text { Weight } \\
(\mathrm{mg} \pm \mathrm{s.d} .)\end{array}$ & $\begin{array}{c}\text { Diameter } \\
(\mathrm{mm} \pm \mathrm{s.d} .)\end{array}$ & $\begin{array}{c}\text { Porosity } \\
(\%)\end{array}$ & $\begin{array}{c}\text { Aspect ratio } \\
(\text { value } \pm \text { s.d. })\end{array}$ & $\begin{array}{c}\text { Crushing strenght } \\
(\mathrm{N} \pm \mathrm{s.d} \text {. })\end{array}$ & $\begin{array}{c}\text { Friability } \\
(\%)\end{array}$ \\
\hline G20-80 & $43.22 \pm 0.34$ & $4.20 \pm 0.13$ & 5.73 & $1.05 \pm 0.04$ & $204 \pm 23$ & 0.42 \\
G20-100 & $40.36 \pm 0.29$ & $4.11 \pm 0.12$ & 6.47 & $1.06 \pm 0.03$ & $165 \pm 17$ & 0.01 \\
G20-100/2 & $33.89 \pm 0.41$ & $3.87 \pm 0.14$ & 6.16 & $1.07 \pm 0.04$ & $170 \pm 15$ & 0.02 \\
G30-30L & $49.43 \pm 0.68$ & $4.26 \pm 0.21$ & 5.59 & $1.02 \pm 0.02$ & $125 \pm 10$ & 0.33 \\
G30-30D & $58.05 \pm 0.79$ & $4.13 \pm 0.16$ & 7.35 & $1.03 \pm 0.02$ & $146 \pm 8$ & 0.13 \\
\hline
\end{tabular}

Table 4: release parameters according to exponential equation (2)

\begin{tabular}{lcccc}
\hline & $\boldsymbol{n}$ & $\begin{array}{c}\text { confidence } \\
\text { interval 95\% }\end{array}$ & $\boldsymbol{k}$ & $\mathbf{R}^{\mathbf{2}}$ \\
\hline UDDMat T30 & 0.832 & 0.061 & 0.229 & 0.989 \\
UDDMat P30 & 0.775 & 0.056 & 0.271 & 0.981 \\
1-layer unit & $*$ & $*$ & $*$ & $*$ \\
2-layer unit & 0.662 & 0.001 & 1.719 & 0.876 \\
3-layer unit & 0.671 & 0.002 & 1.490 & 0.984 \\
4-layer unit & 0.732 & 0.003 & 1.305 & 0.999 \\
NUDDMat G20-80 & 0.756 & 0.025 & 1.190 & 0.999 \\
NUDDMat G20-100 & 1.121 & 0.002 & 0.067 & 0.915 \\
NUDDMat G20-100/2 & 0.993 & 0.002 & 0.123 & 0.940 \\
NUDDMat G30-30L & 1.227 & 0.003 & 0.809 & 0.986 \\
NUDDMat G30-30D & 0.802 & 0.003 & 1.184 & 0.997 \\
\hline
\end{tabular}

*- Not applicable

Raman mapping analysis

374 In order to highlight the drug and polymer distribution throughout the layered formulations, it was deemed 375 interesting to perform imaging analyses by Raman mapping microscopy. The distribution of the drug tracer 376 particles within layered matrices was evaluated on cross-sectioned UDDMat P30 and NUDDMat G30-30D 377 systems. The mapping was performed by assigning false colors to AAP, HPMC (or cellulose), DCP and to the AAP/HPMC ratio after identification of specific peaks of the spectra (Figure 7). Although no HPMC was contained in the seed formulation, quite a strong blue signal was highlighted, because cellulose, namely MCC, was present as the main component. 


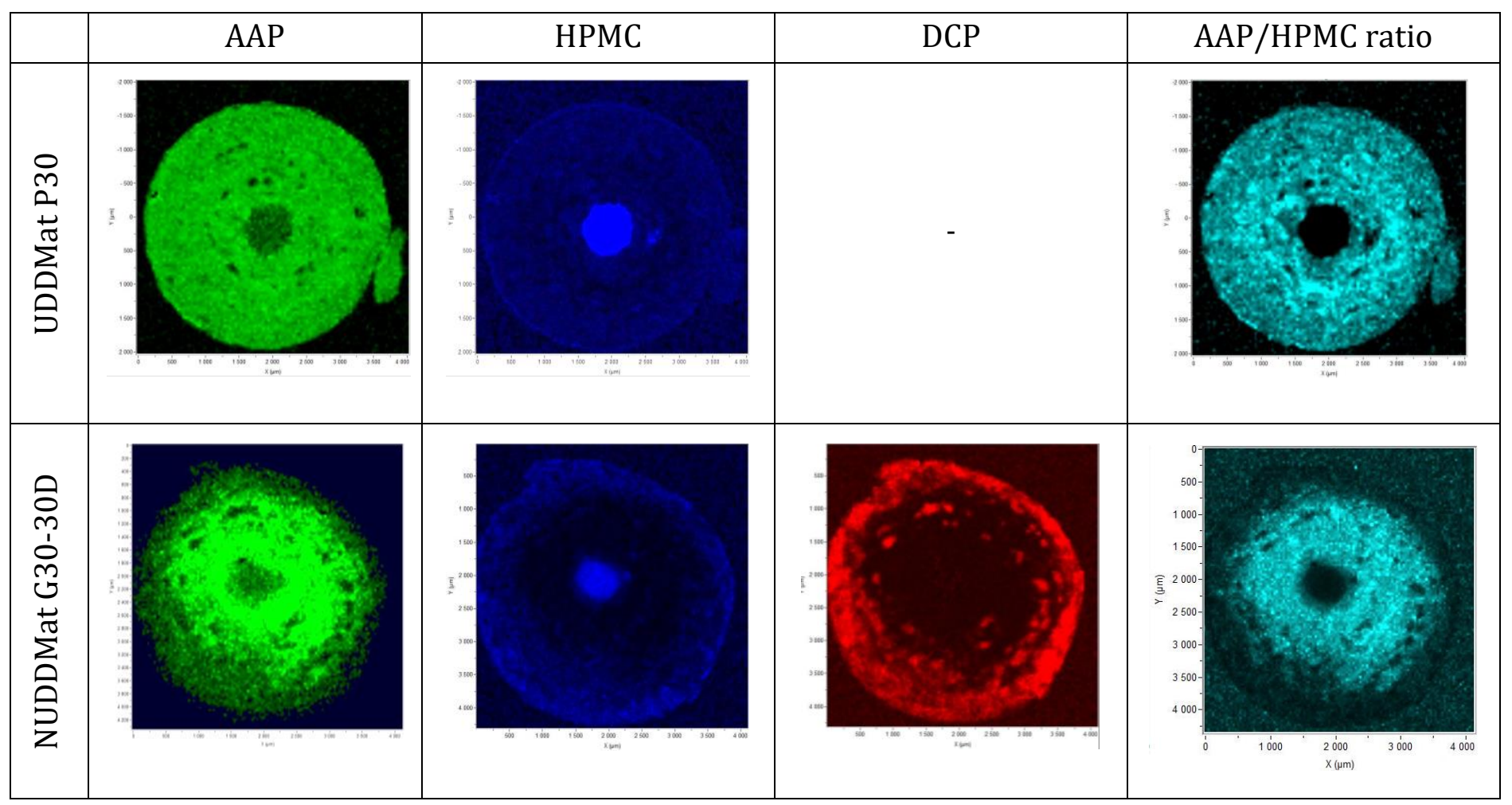

382

383 Figure 7: Raman mapping microscopy images relevant to AAP, HPMC, DCP and AAP/HPMC ratio acquired from

384 cross-sectioned UDDMat P30 and NUDDMat G30-30D systems. Mapping refers to areas of approximately $4000 \mathrm{x}$ $3854000 \mu \mathrm{m}$.

386

387 The color intensity of AAP/HPMC ratio was plotted along the diameter of the cross-sectioned units (Figure 388 8). 
391 a)

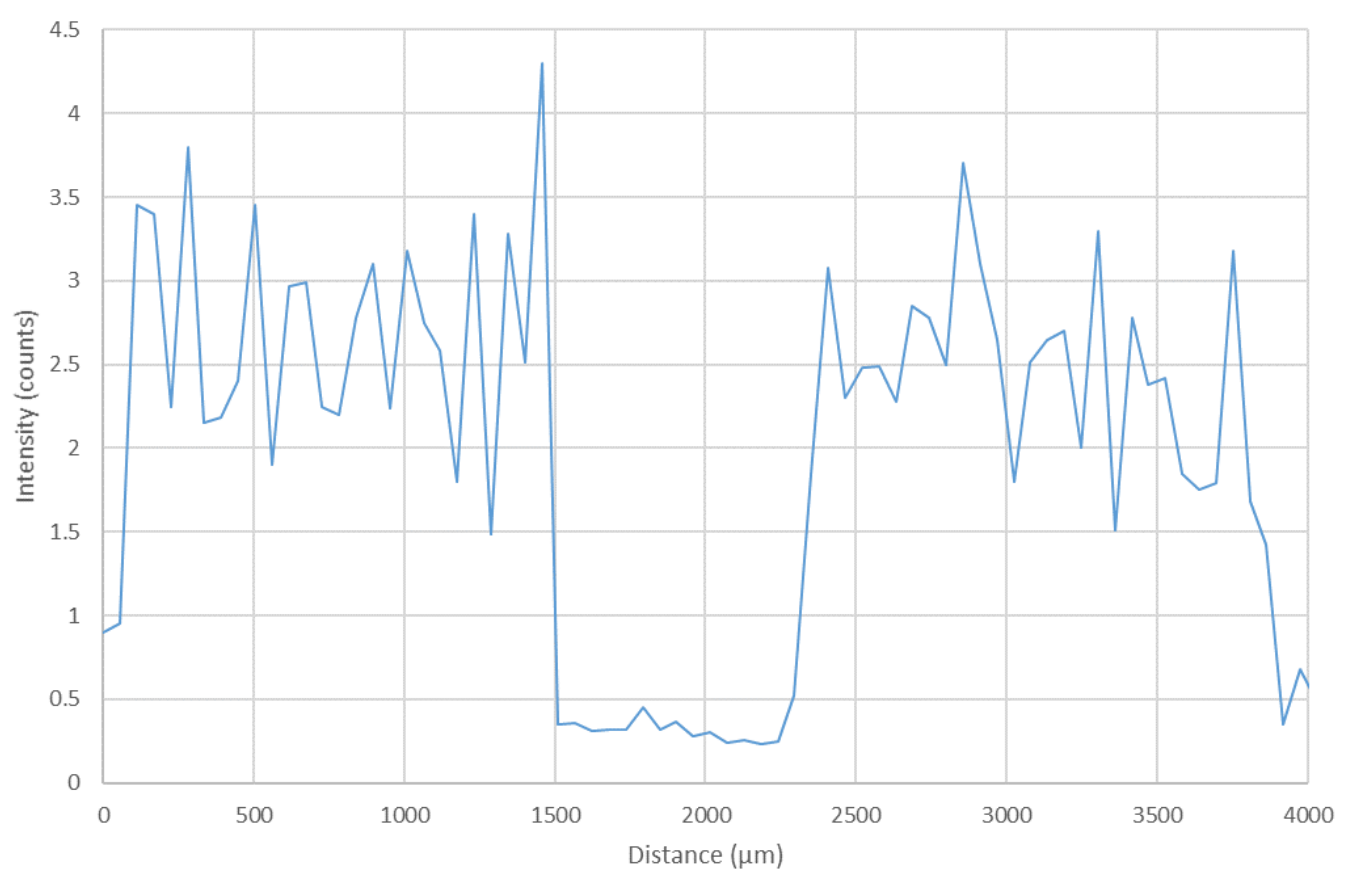

392

393 b)

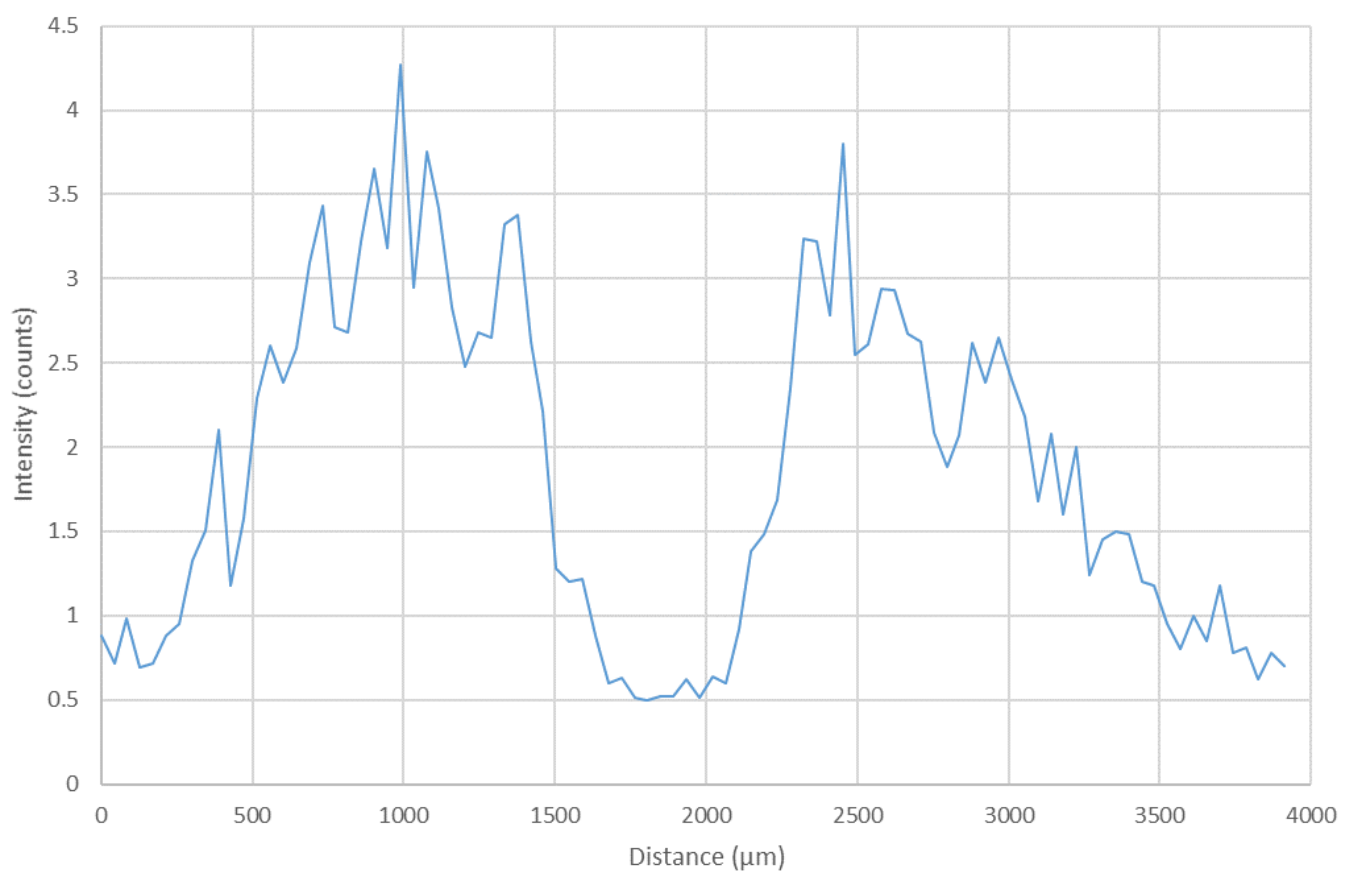
cross-sectioned UDDMat P30 (a) and NUDDMat G30-30D (b) systems 
In the UDDMat P30 system, the AAP/HPMC intensity ratio profile, calculated along one diameter, suggested a uniform concentration of AAP throughout the layered powders and pointed out the sole presence of cellulose in the seed core (Figure 8 a). For NUDDMat G30-30D, a gradient of AAP/HPMC intensity ratio indicated a non-uniform concentration of drug tracer, decreasing from the inside to the outside of the unit. Particularly, a rise towards the center and a steep fall close to the core, devoid of drug, were highlighted, exactly matching the 3D hyperspectral map (Figure $8 \mathrm{~b}$, Figure 9). The lack of a staircase concentration pattern, previously hypothesized from the evaluation of the release curves, seemed to be confirmed by the AAP/HPMC intensity ratio profile.

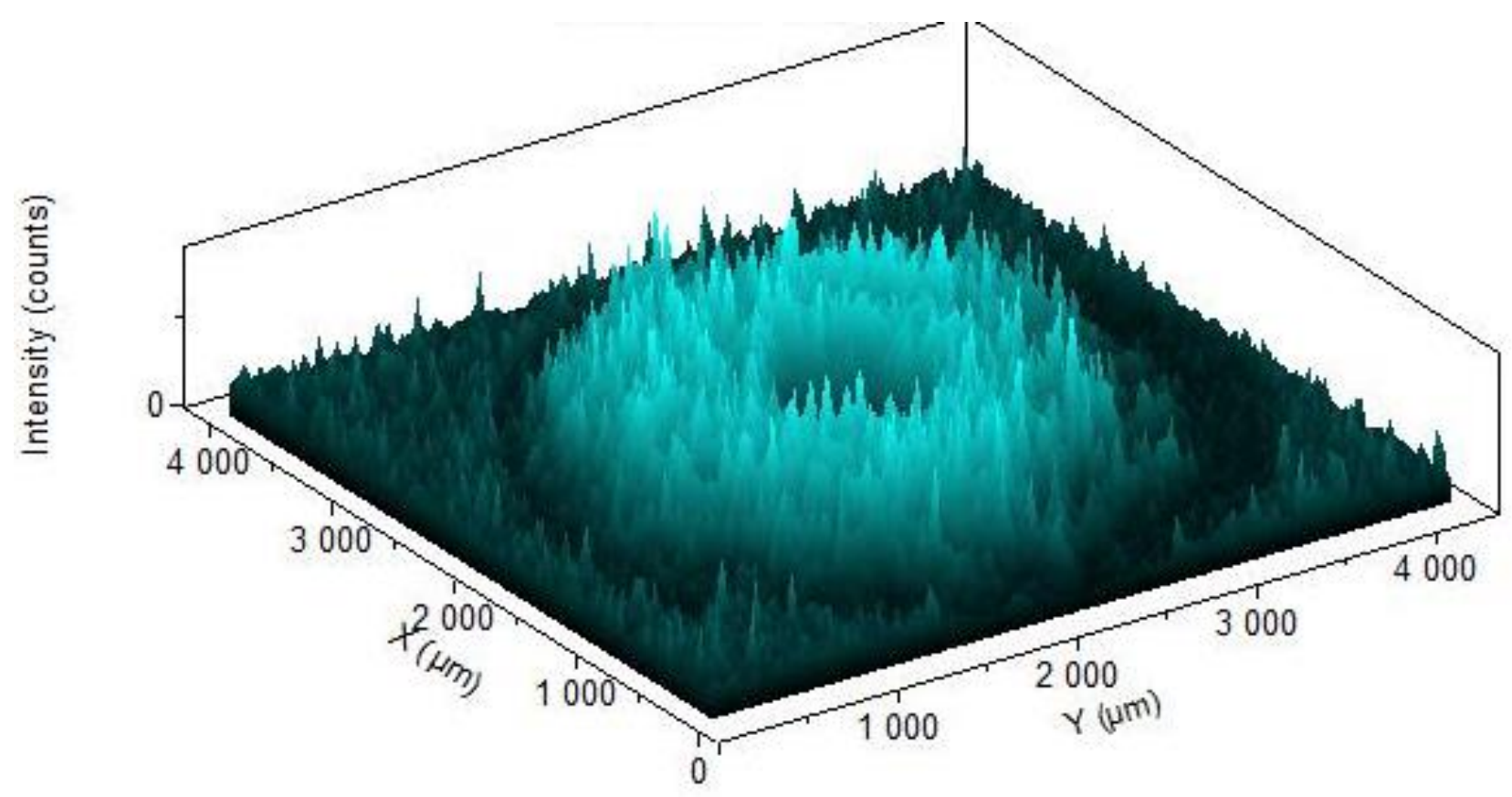

Figure 9: hyperspectral 3D mapping by Raman microscopy relevant to color intensity of AAP/HPMC ratio acquired from a NUDDMat G30-30D system. Mapping refers to areas of approximately $4000 \times 4000 \mu \mathrm{m}$.

\section{CONCLUSIONS}

414 A hydrophilic matrix system intended for zero-order release was designed and fabricated by subsequent 415 deposition onto inert cores of layers having outwards decreasing drug concentrations. For this purpose, the use 416 of powder layering was explored, which offers major advantages because of the small amount of solvents 417 involved and reduced costs as compared with other layering techniques. Spherical units of $4 \mathrm{~mm}$ in diameter 418 were thereby obtained, exhibiting satisfactory physico-technological characteristics. The non-uniform 419 composition of the layered matrix, varying along its cross-sectional axis, was highlighted by Raman mapping analysis. The particular configuration of NUDDMat was demonstrated to reduce the initial burst effect typical 
of hydrophilic matrices, which, as expected, was yielded by a formulation having equal overall composition though uniform drug distribution. Moreover, this configuration proved effective in contrasting other drawbacks that are associated with hydrophilic matrices, such as the lengthening of the diffusional pathway and reduction of the area at the swelling front, both responsible for a progressive decrease in the release rate. The desired zero-order release profiles were thus obtained from the NUDDMat system, as confirmed through Durbin-Watson statistics that pointed out extension of the linear portion of release curves. As regards drug loading, the NUDDMat technology proposed would in principle not differ from classical hydrophilic matrix systems. Actually, based on the NUDDMat G20-100/2 formulation yielding zero-order release, which already contained around $33 \%$ of tracer, drug content higher than $50 \%$ could be achieved. The percentage of polymer could indeed be reduced in the innermost layers provided that in the outer one it is maintained at least in a 20-30\% range, needed to ensure matrix structure formation upon glassy-rubbery transition. The simple design concept of the novel gradient composition matrix and the use of a more advantageous technique as compared with previous attempts reported in the literature make the delivery platform hereby proposed a valuable strategy to improve the performance of hydrophilic matrix systems for prolonged release.

\section{AKNOLEDGEMENTS}

The authors wish to thank Dr Stefano Gay, HORIBA Italia Srl, for Raman mapping analysis and helpful discussion.

\section{REFERENCES}

442 [1] P. Colombo, Swelling-controlled release in hydrogel matrices for oral route, Adv. Drug Deliv. Rev. 11 (1993) 37-57.

444 [2] P. Colombo, A. Gazzaniga, C. Caramella, U. Conte, A. La Manna, In vitro programmable zero-order release drug delivery system, Acta Pharm. Technol. 33 (1987) 15-20.

[3] P. Colombo, U. Conte, A. Gazzaniga, L. Maggi, M.E. Sangalli, N.A. Peppas, A. La Manna, Drug release modulation by physical restrictions of matrix swelling, Int. J. Pharm. 63 (1990) 43-48.

[4] A. Gazzaniga, M.E. Sangalli, U. Conte, C. Caramella, P. Colombo, A. La Manna, On the release mechanism from coated swellable minimatrices, Int. J. Pharm. 91 (1993) 167-171. doi:10.1016/03785173(93)90336-E.

[5] M. Grassi, L. Zema, M.E. Sangalli, A. Maroni, F. Giordano, A. Gazzaniga, Modeling of drug release from partially coated matrices made of a high viscosity HPMC., Int. J. Pharm. 276 (2004) 107-14. doi:10.1016/j.ijpharm.2004.02.016.

[6] C. ju Kim, Compressed Donut-Shaped Tablets with Zero-Order Release Kinetics, Pharm. Res. 12 
(1995) 1045-1048. doi:10.1023/A:1016218716951.

456 [7] M.E. Sangalli, A. Maroni, L. Zema, M. Cerea, U. Conte, A. Gazzaniga, A study on the release

[8] M.E. Sangalli, P. Giunchedi, A. Gazzaniga, U. Conte, Erodible perforated coated matrix for extended

[9] M.E. Sangalli, P. Giunchedi, L. Maggi, U. Conte, A. Gazzaniga, Inert monolithic device with a

[11] P.I. Lee, Novel approach to zero-order drug delivery via immobilized nonuniform drug distribution in

[12] P. de Haan, C.F. Lerk, The megaloporous system: a novel principle for zero-order drug delivery I. In

[13] P.I. Lee, Initial concentration distribution as a mechanism for regulating drug release from diffusion

[15] P. Hildgen, J.N. McMullen, A new gradient matrix: Formulation and characterization, J. Control. Release. 34 (1995) 263-271. doi:10.1016/0168-3659(95)00014-Y.

[16] N. James Chang, K.J. Himmelstein, Dissolution-diffusion controlled constant-rate release from heterogeneously loaded drug-containing materials, J. Control. Release. 12 (1990) 201-212. doi:10.1016/0168-3659(90)90101-X.

[17] D.C. Scott, R.G. Hollenbeck, Design and manufacture of a zero-order sustained-release pellet dosage form through nonuniform drug distribution in a diffusional matrix., Pharm. Res. 8 (1991) 156-61. http://www.ncbi.nlm.nih.gov/pubmed/2023862 (accessed June 24, 2016).

[18] B. Dekyndt, J. Verin, C. Neut, F. Siepmann, J. Siepmann, How to easily provide zero order release of freely soluble drugs from coated pellets, Int. J. Pharm. 478 (2015) 31-38.

[19] A. Melocchi, F. Parietti, G. Loreti, A. Maroni, A. Gazzaniga, L. Zema, 3D Printing by Fused 
Deposition Modeling (FDM) of a swellable/erodible capsular device for oral pulsatile release of drugs, J. Drug Deliv. Sci. Technol. 30 (2015) 360-367. doi:10.1016/j.jddst.2015.07.016.

[20] D.G. Yu, L.-M. Zhu, C.J. Branford-White, X.L. Yang, Three-dimensional printing in pharmaceutics: promises and problems., J. Pharm. Sci. 97 (2008) 3666-90. doi:10.1002/jps.21284.

[21] A. Melocchi, F. Parietti, A. Maroni, A. Foppoli, A. Gazzaniga, L. Zema, Hot-melt extruded filaments based on pharmaceutical grade polymers for 3D printing by fused deposition modeling, Int. J. Pharm. 509 (2016) 255-263. doi:10.1016/j.ijpharm.2016.05.036.

[22] L. Zema, A. Melocchi, A. Maroni, A. Gazzaniga, Three-Dimensional Printing of Medicinal Products and the Challenge of Personalized Therapy, J. Pharm. Sci. 106 (2017) 1697-1705. doi:10.1016/j.xphs.2017.03.021.

[23] M.P. Grosvenor, J.N. Staniforth, The influence of water on electrostatic charge retention and dissipation in pharmaceutical compacts for powder coating, Pharm. Res. 13 (1996) 1725-1729. doi:10.1023/A:1016409227565.

[24] A. Maroni, L. Zema, M. Cerea, A. Foppoli, L. Palugan, A. Gazzaniga, Erodible drug delivery systems for time-controlled release into the gastrointestinal tract, J. Drug Deliv. Sci. Technol. 32 (2016) 229 235. doi:10.1016/j.jddst.2015.10.001.

[25] A. Maroni, L. Zema, G. Loreti, L. Palugan, A. Gazzaniga, Film coatings for oral pulsatile release., Int. J. Pharm. 457 (2013) 362-71. doi:10.1016/j.ijpharm.2013.03.010.

[26] D. Sauer, M. Cerea, J. Dinunzio, J. McGinity, Dry powder coating of pharmaceuticals: A review, Int. J. Pharm. 457 (2013) 488-502.

[27] A. Foppoli, A. Maroni, M. Cerea, L. Zema, A. Gazzaniga, Dry coating of solid dosage forms: an overview of processes and applications, Drug Dev. Ind. Pharm. (2017) 1-14. doi:10.1080/03639045.2017.1355923.

[28] M.J. O’Neil, The Merck Index - An Encyclopedia of Chemicals, Drugs, and Biologicals, 2013. doi:10.1007/s13398-014-0173-7.2.

[29] F. Giordano, A. Rossi, R. Bettini, A. Savioli, A. Gazzaniga, C. Novák, Thermal Behavior of Paracetamol- Polymeric Excipients Mixtures, J. Therm. Anal. Calorim. 68 (2002) 575-590.

[30] A. Rossi, A. Savioli, M. Bini, D. Capsoni, V. Massarotti, R. Bettini, A. Gazzaniga, M.E. Sangalli, F. Giordano, Solid-state characterization of paracetamol metastable polymorphs formed in binary mixtures with hydroxypropylmethylcellulose, Thermochim. Acta. 406 (2003) 55-67. doi:10.1016/S0040-6031(03)00227-2.

[31] Colorcon, Using METHOCEL Cellulose Ethers for Controlled Release of Drugs in Hydrophilic 
Matrix Systems, 2000. (2000). http://www.colorcon.com/literature/marketing/mr/Extended Release/METHOCEL/English/hydroph_matrix_broch.pdf (accessed June 24, 2016).

[32] M. Qi, P. Wang, D. Wu, A novel pH- and time-dependent system for colonic drug delivery., Drug Dev. Ind. Pharm. 29 (2003) 661-7. doi:10.1081/DDC-120021315.

[33] C.-Y. Wu, S.M. Best, A.C. Bentham, B.C. Hancock, W. Bonfield, A simple predictive model for the tensile strength of binary tablets, Eur. J. Pharm. Sci. 25 (2005) 331-336. doi:10.1016/j.ejps.2005.03.004.

[34] T.A. Meyer, Novel Determination of Powder Mixing Qualities and Study of Dry Coated Particles, (2008). http://edoc.unibas.ch/791/1/DissB_8366.pdf (accessed August 12, 2016).

[35] R.J. Roberts, R.C. Rowe, The effect of punch velocity on the compaction of a variety of materials, J. Pharm. Pharmacol. 37 (1985) 377-384. doi:10.1111/j.2042-7158.1985.tb03019.x.

[36] P.J. Marsac, S.L. Shamblin, L.S. Taylor, Theoretical and Practical Approaches for Prediction of Drug-Polymer Miscibility and Solubility, Pharm. Res. 23 (2006) 2417-2426. doi:10.1007/s11095006-9063-9.

[37] C. Sun, True density of microcrystalline cellulose, J. Pharm. Sci. (2005). doi:10.1002/jps.20459.

[38] United States Pharmacopeia Convention, General Chapters: <1010>Analytical Data-Interpretation and Treatment, in: USP 40 United States Pharmacop. - NF 35 Natl. Formul., 2017: p. 836.

[39] D. Chambers, G. Kelly, G. Limentani, A. Lister, K.R. Lung, E. Warner, Analytical method equivalence - An acceptable analytical practice, Pharm. Technol. (2005) 64-80. www.pharmtech.com (accessed October 11, 2017).

[40] Administration Centre For Drug US Department of Health and Human Services Food and Drug, CDER Evaluation And Research, Guidance for industry dissolution testing of immediate release solid oral dosage forms, 1997. http://www.fda.gov/downloads/Drugs/.../Guidances/ucm070246.pdf\%5C.

[41] G.W. Sinclair, N.A. Peppas, Analysis of non-fickian transport in polymers using simplified exponential expressions, J. Memb. Sci. 17 (1984) 329-331.

[42] P. Colombo, U. Conte, C. Caramella, A. Gazzaniga, A. La Manna, Compressed polymeric minimatrices for drug release control, J. Control. Release. 1 (1985) 283-289. doi:10.1016/01683659(85)90004-5.

[43] R.W. Korsmeyer, R. Gurny, E. Doelker, P. Buri, N.A. Peppas, Mechanisms of solute release from porous hydrophilic polymers, Int. J. Pharm. 15 (1983) 25-35. doi:10.1016/0378-5173(83)90064-9.

[44] J. Durbin, G.S. Watson, Testing for serial correlation in least squares regression. I., Biometrika. 37 (1950) 409-28. http://www.ncbi.nlm.nih.gov/pubmed/14801065 (accessed June 24, 2016). 
551 [45] H. van der Voet, P. de Haan, D.A. Doornbos, The use of the Durbin-Watson statistic for testing the validity of kinetic models for dissolution, Int. J. Pharm. 14 (1983) 291-298. doi:10.1016/03785173(83)90101-1.

554

555

556

557

558

559

560

561

562

563

564

565

566

567

568

569

[46] S. Lakio, B. Vajna, I. Farkas, H. Salokangas, G. Marosi, J. Yliruusi, Challenges in detecting magnesium stearate distribution in tablets., AAPS PharmSciTech. 14 (2013) 435-44. doi:10.1208/s12249-013-9927-3.

[47] C. Nastruzzi, R. Cortesi, E. Esposito, A. Genovesi, A. Spadoni, C. Vecchio, E. Menegatti, Influence of formulation and process parameters on pellet production by powder layering technique, AAPS PharmSciTech. 1 (2000) 14-25. doi:10.1208/pt010209.

[48] I. Tomuta, S.E. Leucuta, Use of experimental design for identifying the most important formulation and technological variables in pelletization by powder layering, J. Drug Deliv. Sci. Technol. 14 (2004) 215-221. doi:10.1016/S1773-2247(04)50103-X.

[49] R. Bettini, P. Colombo, G. Massimo, P.L. Catellani, T. Vitali, Swelling and drug release in hydrogel matrices: polymer viscosity and matrix porosity effects, Eur. J. Pharm. Sci. 2 (1994) 213-219. doi:10.1016/0928-0987(94)90025-6.

[50] P.L. Ritger, N.A. Peppas, A simple equation for description of solute release I. Fickian and nonfickian release from non-swellable devices in the form of slabs, spheres, cylinders or discs, J. Control. Release. 5 (1987) 23-36. doi:10.1016/0168-3659(87)90034-4. 